\section{(2) OPEN ACCESS}

\title{
Interphysician agreement on subclassification of myocardial infarction
}

\author{
Anton Gard, ${ }^{1,2}$ Bertil Lindahl, ${ }^{1,2}$ Gorav Batra, ${ }^{1,2}$ Nermin Hadziosmanovic, ${ }^{1,2}$ \\ Marcus Hjort, ${ }^{1,2}$ Karolina Elisabeth Szummer, ${ }^{3,4}$ Tomasz Baron $^{1,2}$
}

${ }^{1}$ Department of Medical Sciences, Cardiology, Uppsala University, Uppsala, Sweden ¿Uppsala Clinical Research Center, Uppsala University, Uppsala, Sweden

${ }^{3}$ Department of Medicine, Huddinge, Section of Cardiology, Karolinska Institute, Uppsala, Sweden

${ }^{4}$ Department of Cardiology, Karolinska University Hospital, Stockholm, Sweden

\section{Correspondence to} Mr Anton Gard, Department of Cardiology, Uppsala Clinical Research Center, Uppsala 75185, Sweden; gardanton@ gmail.com

Received 6 September 2017 Revised 29 December 2017 Accepted 2 January 2018 Published Online First 16 February 2018
Check for updates

To cite: Gard A, Lindahl B, Batra $\mathrm{G}$, et al. Heart

2018;104:1284-1291.

\section{ABSTRACT}

Objective The universal definition of myocardial infarction (MI) differentiates MI due to oxygen supply/demand mismatch (type 2) from Ml due to plaque rupture (type 1) as well as from myocardial injuries of non-ischaemic or multifactorial nature. The purpose of this study was to investigate how often physicians agree in this classification and what factors lead to agreement or disagreement. Methods A total of 1328 patients diagnosed with MI at eight different Swedish hospitals 2011 were included. All patients were retrospectively reclassified into different $\mathrm{Ml}$ or myocardial injury subtypes by two independent specially trained physicians, strictly adhering to the third universal definition of MI.

Results Overall, there was a moderate interobserver agreement with a kappa coefficient ( $\mathrm{\kappa}$ ) of 0.55 in this classification. There was substantial agreement when distinguishing type $1 \mathrm{MI}$ (к: 0.61), compared with moderate agreement when distinguishing type $2 \mathrm{Ml}$ ( $\kappa: 0.54)$. In multivariate logistic regression analyses, ST elevation MI $(\mathrm{P}<0.001)$, performed coronary angiography $(P<0.001)$ and larger changes in troponin levels $(P=0.023)$ independently made the physicians agree significantly more often, while they disagreed more often with symptoms of dyspnoea $(\mathrm{P}<0.001)$, higher systolic blood pressure $(P=0.001)$ and higher $C$ reactive protein levels on admission $(P=0.016)$.

Conclusion Distinguishing Ml types is challenging also for trained adjudicators. Although strictly adhering to the third universal definition of $\mathrm{Ml}$, differentiation between type $1 \mathrm{Ml}$, type $2 \mathrm{Ml}$ and myocardial injury only gave a moderate rate of interobserver agreement. More precise and clinically applicable criteria for the current classification, particularly for type $2 \mathrm{MI}$ diagnosis, are urgently needed.

\section{INTRODUCTION}

The development of increasingly more sensitive biomarkers for detecting myocardial injury and necrosis over the last decades has required several updates and revisions of the definition of myocardial infarction (MI). The universal definition of MI, published in 2007 and revised in 2012, has introduced five clinical subtypes of MI depending on the underlying pathophysiological mechanism leading to the ischaemic necrosis. ${ }^{1}$ All MI subtypes have in common that the myocardial necrosis is caused by myocardial ischaemia. However, while type $1 \mathrm{MI}$ is caused by a local thromboembolic formation in the coronary artery due to plaque rupture, ulceration, fissuring or dissection, in type $2 \mathrm{MI}$ other conditions than coronary artery disease (CAD) contributes to an ischaemic imbalance in cardiac oxygen supply and demand. Apart from the ischaemic necrosis in MI, myocardial injury and necrosis may occur in other settings where inflammation, toxicity, trauma or mechanical stress is causing or contributing to the injury. While in some conditions, such as in myocarditis or trauma, the myocardial injury is non-ischaemic, in other conditions (such as heart failure or sepsis) the myocardial injury may be of a multifactorial nature where the significance of a possible ischaemic component is difficult to decide. $^{2}$ Distinguishing type 2 MI from type 1 MI and especially from multifactorial myocardial injuries constitutes a clinical challenge. The interpretation of this classification differs considerably between different studies indicating that the clinical criteria for this classification are not well defined. ${ }^{3-5}$ Therefore, the aims of this study were (1) to evaluate how often physicians agree in distinguishing type $1 \mathrm{MI}$, type $2 \mathrm{MI}$ and multifactorial myocardial injury, when adhering strictly to the classification presented by the third universal definition of $M I$ and (2) to elucidate which clinical variables that affect agreement or disagreement between reviewers in this classification.

\section{MATERIALS AND METHODS Study population}

A total of 1328 consecutive patients treated at eight Swedish hospitals of different size of the year 2011 who were diagnosed with acute MI (ICD code I.21) at discharge were included in the study. The inclusion was stratified according to whether the patients were registered in the national register for MI (Swedish Web-system for Enhancement and Development of Evidence-based care in Heart disease Evaluated According to Recommended Therapies-SWEDEHEART) or not. ${ }^{6}$ The aim was to include the first 100 patients of year 2011 at each hospital registered in the SWEDEHEART registry and the first up to 100 at each hospital not registered in the SWEDEHEART registry $(<100$ patients were diagnosed with MI outside the registry in some hospitals). Mainly patients with MI treated in a cardiology department are included in the SWEDEHEART registry; patients diagnosed with MI at other departments are less often included. The registry had during the study period a completeness of $82 \%$ of all MIs diagnosed in Sweden. ${ }^{7}$ Eight patients were excluded because they had not got the ICD code I.21 at discharge 
SWEDEHEART registered

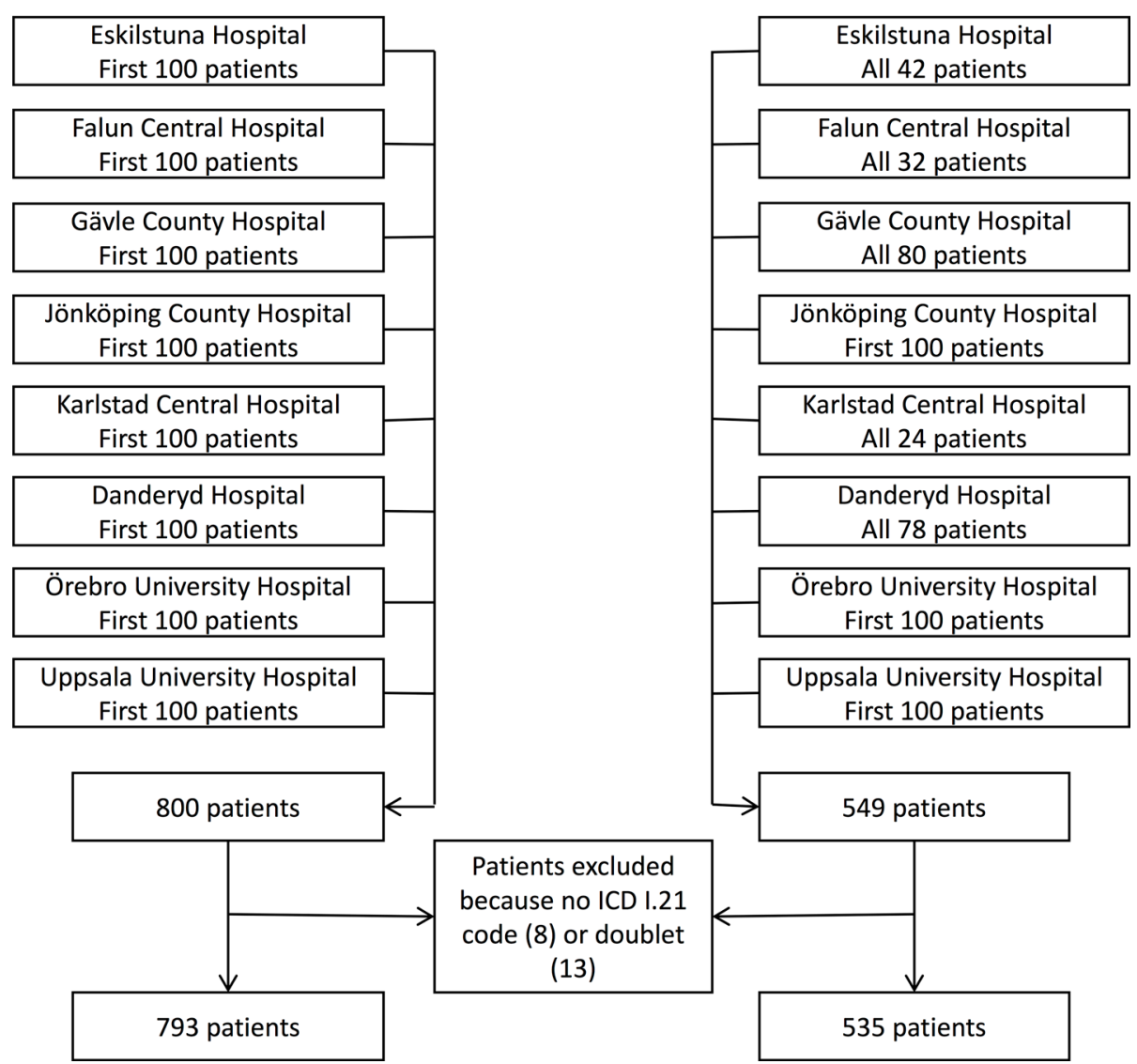

Figure 1 Patient selection process. ICD, International Classification of Diseases; ICD 1.21, ICD code for acute myocardial infarction.

and 13 patients were excluded from the non-registered group since they also appeared among registered patients. This made the final number of 793 patients registered in SWEDEHEART and 535 patients not registered (figure 1).

\section{Collection of data}

Using a prespecified case report form, detailed patient information (such as age, sex, comorbidities, medications on admission, clinical parameters, laboratory results, ECGs, results from invasive and non-invasive investigations, treatments in hospital and medications on discharge) was retrospectively collected from the electronic patient records of each hospital.

\section{Troponin assays}

Different cardiac troponin (cTn) assays with different reference values were used at the different hospitals in this study. Reviewers had access to all original cTn values, reference values and time of sampling during the classification process, while all $c T n$ results presented on a group level have been standardised by dividing the cTn level with the 99th percentile upper reference limit of the assay used.

\section{Classification process}

In each patient, the MI diagnosis was retrospectively validated and all patients were classified as MI type 1-5, multifactorial myocardial injury or non-ischaemic myocardial injury by two independent specially trained physicians using a prespecified form based on the third universal definition of MI (see online supplementary file). Rate of agreement was analysed between the initial two reviewers. For group comparison analyses, a final diagnosis for each patient was decided by majority rule, using a third physician, and in a few cases a fourth physician, in cases of disagreement between the initial two reviewers.

\section{Statistics}

Groups were compared using Mann-Whitney U test for non-normally distributed numerical variables, presented as medians with 25th and 75th percentiles; Student's t-test for normally distributed numerical variables, presented as means with SD; and Pearson's $\mathrm{X}^{2}$ test for categorical variables, presented as numbers with percentages.

Agreement between the initial two reviewers was calculated using Cohen's Kappa statistics.

A multivariate logistic regression model with backward selection was used to analyse if clinical variables were independently associated with agreement or disagreement between the initial two reviewers. Clinical variables that differed significantly between cases of agreement and cases of disagreement in a univariate model were selected. Variables with more than 25\% missing cases were excluded (online supplementary table S1). Four variables-age, sex, performed coronary angiography and performed echocardiography - were forced into the final multivariate model regardless of the outcome in the univariate model or the backward selection. All non-normally distributed continuous variables were logarithmised and all continuous variables were divided by their SD.

Further, to analyse the degree of agreement for each significant variable in the multivariate analysis, a stratified analysis 
SWEDEHEART (n 793)

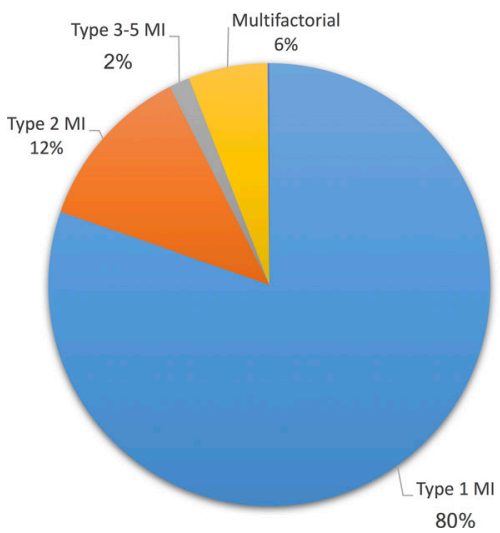

Not-SWEDEHEART (n 535)

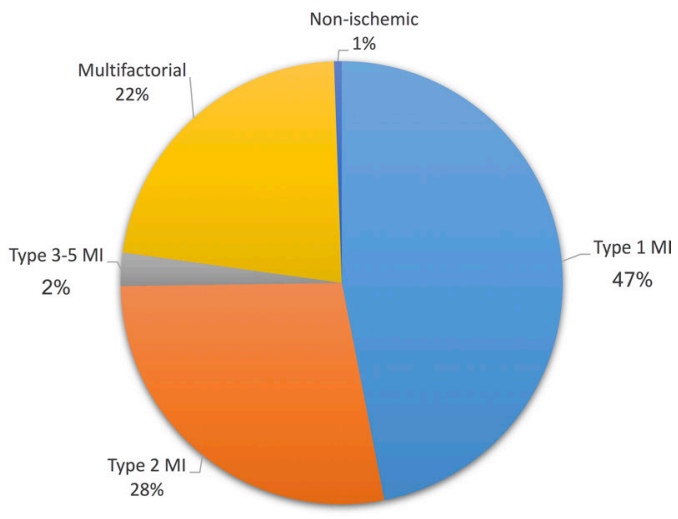

Figure 2 Prevalence of $\mathrm{MI}$ and myocardial injury subtypes among patients registered and not registered in SWEDEHEART. MI, myocardial infarction; SWEDEHEART, Swedish Web-system for Enhancement and Development of Evidence-based care in Heart disease Evaluated According to Recommended Therapies.

with kappa statistics was performed. Numeric variables were stratified based on their median value.

All analyses were performed using IBM SPSS Statistics V.24.0 (SPSS, IBM, Armonk, New York, USA).

\section{RESULTS}

After the validation and classification process, an MI diagnosis was confirmed in 1159 patients (87.3\%). A total of 888 patients were classified as type $1 \mathrm{MI}, 246$ as type $2 \mathrm{MI}, 9$ as type $3 \mathrm{MI}$ and 16 as type $4 \mathrm{a}$ or $4 \mathrm{~b}$ MI. In 169 cases, the MI diagnosis was considered incorrect with 165 patients re-classified to a multifactorial myocardial injury and 4 patients to a non-ischaemic myocardial injury. The prevalence of MI types and myocardial injury differed considerably between patients registered and not registered in SWEDEHEART (figure 2).

Compared with type $1 \mathrm{MI}$, patients with type $2 \mathrm{MI}$ were older, more often female, had more cardiovascular or other comorbidities, were significantly less often investigated with coronary angiography and less often invasively or pharmacologically treated to address CAD. In contrast, only a few differences were seen between patients with type $2 \mathrm{MI}$ and multifactorial myocardial injury (table 1). The inhospital mortality rate was higher in patients with multifactorial myocardial injury compared with those with both type $1 \mathrm{MI}$ and type $2 \mathrm{MI}(23.6 \%$ vs $9.7 \%$ and $11.0 \%$, respectively).

\section{Triggering mechanisms}

The most common triggering mechanisms causing type $2 \mathrm{MI}$ were tachyarrhythmia, anaemia and respiratory failure and the by far most common underlying condition associated with multifactorial myocardial injury was heart failure, followed by renal failure and sepsis (figure 3).

\section{Classification agreement analyses}

Overall, agreement between the initial two reviewers was reached in 1022 cases $(77 \%)$ with a Kappa value $(\kappa)$ of 0.55 (95\% CI 0.51 to 0.59 ), representing a moderate rate of agreement (table 2).

The two initial reviewers agreed in $85.7 \%$ (716/888), $64.2 \%$ $(158 / 246)$ and $55.2 \%(91 / 165)$ of the cases adjudicated as type 1 $\mathrm{MI}$, type $2 \mathrm{MI}$ and multifactorial injury, respectively. As shown in online supplementary tables S2-S4, when distinguishing type 1 MI there was a substantial rate of agreement between reviewers (к: $0,61,95 \%$ CI0.57 to 0.66 ) compared with a moderate rate of agreement when distinguishing type $2 \mathrm{MI}(\kappa: 0.54,95 \% \mathrm{CI} 0.48$ to 0.60 ) and multifactorial myocardial injury ( $\kappa: 0.48,95 \% \mathrm{CI}$ 0.41 to 0.55 ).

Analysed in a multivariate logistic regression model, ST elevation MI, current smoking, performed coronary angiography and a more dynamic cTn pattern all independently made reviewers agree significantly more often, while agreement decreased significantly with higher systolic blood pressure, symptoms of dyspnoea, higher CRP levels on admission and a history of stroke or transient ischaemic attack (TIA) (figure 4, online supplementary table S5). Stratified kappa analyses showed kappa values in line with the $\%$ agreement in the different strata, except in the stratum of patients with ST elevation MI where the kappa value fell to a fair level $(\kappa: 0,29)$ despite an increase in \% agreement (online supplementary table S5).

\section{DISCUSSION}

This is the first study to investigate in detail how physicians agree adhering to the MI and myocardial injury classification presented in third universal definition of MI. Even for specially trained physicians strictly adhering to this classification, it is challenging especially to distinguish type $2 \mathrm{MI}$ and multifactorial myocardial injury where reviewers reached lower grade of agreement than when distinguishing type $1 \mathrm{MI}$. This is also the first study to investigate which clinical variables or investigations influence the grade of agreement, giving suggestions to where the guidelines should be improved.

While differences in group characteristics between type 1 and type $2 \mathrm{MI}$ are well documented, ${ }^{3-12}$ less is known about how patients with type $2 \mathrm{MI}$ and multifactorial myocardial injury differ from each other. In line with some previous studies, patients with type $2 \mathrm{MI}$ and multifactorial myocardial injury in this study were similar regarding age, sex and comorbidities, ${ }^{5} 1314$ and patients with type $2 \mathrm{MI}$ had higher peak cTn levels. ${ }^{13}{ }^{14} \mathrm{~A}$ higher incidence of chest pain in the type 2 MI group compared with the myocardial injury group was also observed. A high heart rate and a low haemoglobin level also predisposed to type 2 MI diagnose, where tachyarrhythmia and anaemia were the most common provoking mechanisms. ${ }^{13} 14$

Generally, all studies on type 2 MI claim to base on the classification in the third universal definition of MI, but there are still vast differences in how the type $2 \mathrm{MI}$ concept is interpreted, 
Table 1 Comparison in baseline characteristics, clinical parameters, investigations and treatment between type $1 \mathrm{MI}$, type $2 \mathrm{Ml}$ and multifactorial myocardial injury

\begin{tabular}{|c|c|c|c|c|c|}
\hline & Type 1 MI & Type 2 MI & $\begin{array}{l}\text { Multifactorial myocardial } \\
\text { injury }\end{array}$ & p $T 1$ vs $T 2$ & $p T 2$ vs $M$ \\
\hline Total number & 888 & 246 & 165 & & \\
\hline Age, years, mean (SD) & $73.0(12.9)$ & $79.5(11.2)$ & $79.9(10.8)$ & $<0.001$ & ns \\
\hline \multicolumn{6}{|l|}{ Risk factors and medical history, $\mathrm{n}(\%)$} \\
\hline Current smoking & $175(19.7)$ & $26(10.6)$ & $12(7.3)$ & 0.001 & ns \\
\hline Hyperlipidaemia & $230(25.9)$ & $40(16.3)$ & $36(21.8)$ & 0.002 & ns \\
\hline Prior Ml & $255(28.7)$ & $97(39.4)$ & $63(38.2)$ & 0.001 & ns \\
\hline Known heart failure & $103(11.6)$ & $62(25.2)$ & $48(29.1)$ & $<0.001$ & ns \\
\hline Chronic kidney disease & $56(6.3)$ & $39(15.9)$ & $28(15.8)$ & $<0.001$ & ns \\
\hline \multicolumn{6}{|l|}{ Symptoms at admission, n (\%) } \\
\hline Heart rate & 78/846 (67-90) & $92 / 236(78-120)$ & $88 / 157(70-100)$ & $<0.001$ & $<0.001$ \\
\hline \multicolumn{6}{|l|}{ Treating department, n (\%) } \\
\hline Cardiology department & $716(80.6)$ & $132(53.7)$ & $60(36.4)$ & $<0.001$ & 0.001 \\
\hline \multicolumn{6}{|l|}{ Laboratory results, median (IQR)* } \\
\hline Tn maximum level, standarlised & $69.09(15.0-310.0)$ & $28.15(9.4-28.1)$ & $8.93(3.8-38.3)$ & $<0.001$ & $<0.001$ \\
\hline Tn dynamic, $\%$, standarlised $t$ & $412 \%(49 \%-240 \%)$ & $346 \%(80 \%-226 \%)$ & $60 \%(12 \%-273 \%)$ & ns & $<0.001$ \\
\hline CRP maximum level, mg/L & $5.00(2.5-27.0)$ & $29.50(5.0-93.0)$ & $41.50(5.0-142.5)$ & $<0.001$ & ns \\
\hline Creatinine maximum level, $\mu \mathrm{mol} / \mathrm{L}$ & $88.00(73.0-114.0)$ & $104.00(81.5-152.5)$ & $103.00(77.0-154.7)$ & $<0.001$ & ns \\
\hline Haemoglobin on admission, $\mathrm{g} / \mathrm{L}$ & $138.00(126.0-148.0)$ & $121.0(107.7-137.0)$ & $128.0(118.0-140.0)$ & $<0.001$ & 0.002 \\
\hline \multicolumn{6}{|l|}{ Investigations in hospital, n (\%) } \\
\hline Statins & $664(82.8)$ & $110(50.2)$ & $67(53.2)$ & $<0.001$ & ns \\
\hline Anticoagulants & $53(6.6)$ & $36(16.4)$ & $22(17.5)$ & $<0.001$ & ns \\
\hline
\end{tabular}

${ }^{*}>95 \%$ tested in all subtypes.

$\dagger((c T n$ maximum level-cTn minimum level)/cTn minimum level) $x 100$.

$\mathrm{CRP}, \mathrm{C}$ reactive protein; $\mathrm{CTn}$, cardiac troponin; $\mathrm{M}$, multifactorial myocardial injury; MI, myocardial infarction; $\mathrm{CA}$, coronary artery; PCI, percutaneous coronary intervention; RAAS, renin angiotensin aldosterone system.

especially in relation to myocardial injury. While in some studies, all patients without type $1 \mathrm{MI}$ but with elevated cTn are called type $2 \mathrm{MI},{ }^{4}$ in other studies only myocardial injury caused by the mechanisms that may lead to cardiac oxygen supply/demand mismatch presented in third universal definition of $M I$ are classified as type $2 \mathrm{MI}^{3}$ Some studies even uses specific cut-off values for these mechanisms (eg, tachyarrhythmia $>120$ beats per minute) to further sharpen the type 2 MI diagnose. ${ }^{3} 1516$ A condition causing much ambiguity is sepsis. In many studies, sepsis is reported as one of the most common causes of type 2 MI. ${ }^{515} 16$ However, according to the third universal definition of $M I$, the myocardial injury seen in sepsis is not solely related to myocardial ischaemia but rather of a multifactorial nature and should not routinely be classified as type $2 \mathrm{MI}^{2}$ On the other hand, septic shock is postulated as a triggering mechanism of type $2 \mathrm{MI}$; however, in clinical practice, differentiation between those two conditions may be difficult. Heart failure is another condition causing trouble in this classification. In this study, 78 patients out of 1326 patients had incorrectly received MI diagnose based on elevated cTn levels secondary to heart failure. Elevated cTn in acute heart failure should always raise the suspicion of a type $1 \mathrm{MI}$, and also indicate an increased wall stress causing either secondary ischaemia or apoptosis and, experimentally demonstrated, also proteolysis of the cardiac contractile apparatus. ${ }^{17}$ Cardiac troponins are poor diagnostic markers for an ischaemic versus non-ischaemic aetiology in heart failure. ${ }^{18}$ In absence of clinical evidence of ischaemia, the myocardial injury should be regarded as multifactorial. ${ }^{2}$ Since both chest discomfort may occur and abnormal ECG findings frequently occur in patients with acute heart failure, assessing the nature of a myocardial injury in this condition constitutes a great clinical challenge. ${ }^{19}$ 

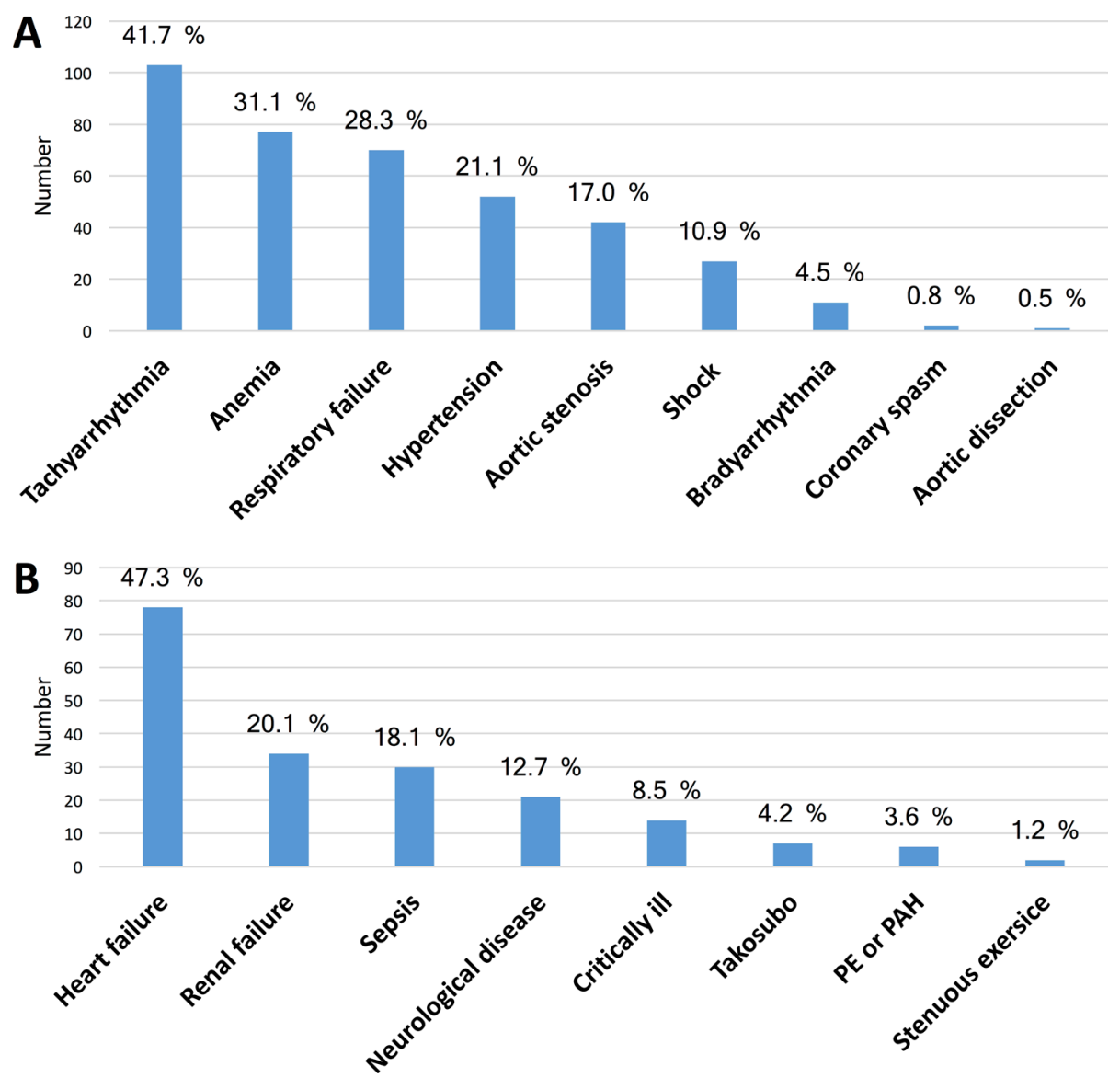

Figure 3 Underlying conditions associated with a secondary myocardial injury. (A) Triggering mechanisms causing an ischaemic imbalance in type 2 myocardial infarction. (B) Conditions associated with a multifactorial myocardial injury. PE, pulmonary embolism; PAH, pulmonary arterial hypertension.

When evaluating agreement between observers, kappa statistics rather than raw (percentage) agreement is preferred since it takes chance into account. ${ }^{20}$ Overall, there was a moderate rate of agreement between reviewers with a $\kappa$ value of 0.55 when classifying the patients into MI and myocardial injury subtypes in this study. This is in contrast to the almost perfect agreement ( $\kappa: 0.92)$ between reviewers in a recent study. ${ }^{5}$ There are some circumstances that may explain part of the difference in $\kappa$ values. In their study, the pathophysiological mechanisms leading to the myocardial injury were not taken into account when differentiating between type $2 \mathrm{MI}$ and myocardial injury. For example, cTn elevation in pericarditis, which is clearly not caused by ischaemia, was classified as type $2 \mathrm{MI}$ as well as cTn elevation in renal failure, neurological diseases, sepsis and heart failure where the myocardial injury is multifactorial according to the third universal definition of $M I^{2}{ }^{2}$ Furthermore, all patients included were initially diagnosed with MI in this study. This naturally means that the patients with myocardial injury in this study resembled patients with MI more than patients with myocardial injury in general.

A high rate of agreement between physicians when distinguishing type 1 from type $2 \mathrm{MI}$ is desirable, since the handling and treatment of patients with these MI types clearly differs. The clinical importance of distinguishing type 2 MI from multifactorial myocardial injury is however less obvious, since there are no guidelines motivating different treatment strategies between these two conditions.

The results from the multivariate logistic regression analysis and the stratified kappa analysis show that performing a coronary angiography helps physicians in classifying a MI or myocardial injury by making the assessment more reproducible, and therefore raising the rate of agreement between reviewers.

Table 2 Comparison of MI and myocardial injury classification between the initial two reviewers ( $\kappa: 0.55$; overall agreement: 77.0\%)

\begin{tabular}{|c|c|c|c|c|c|c|}
\hline \multirow[t]{2}{*}{ First reviewer } & \multicolumn{6}{|c|}{ Second reviewer } \\
\hline & Ml type 1 & MI type 2 & MI types 3-5 & Multifactorial & Non-ischaemic & Total \\
\hline MI type 1 & 716 & 69 & 11 & 46 & 0 & 887 \\
\hline Ml type 2 & 53 & 158 & 2 & 32 & 0 & 245 \\
\hline Ml types 3-5 & 12 & 2 & 10 & 4 & 0 & 28 \\
\hline Multifactorial & 38 & 28 & 1 & 91 & 1 & 159 \\
\hline Non-ischaemic & 2 & 2 & 0 & 3 & 2 & 9 \\
\hline
\end{tabular}

$\mathrm{Ml}$, myocardial infarction. 


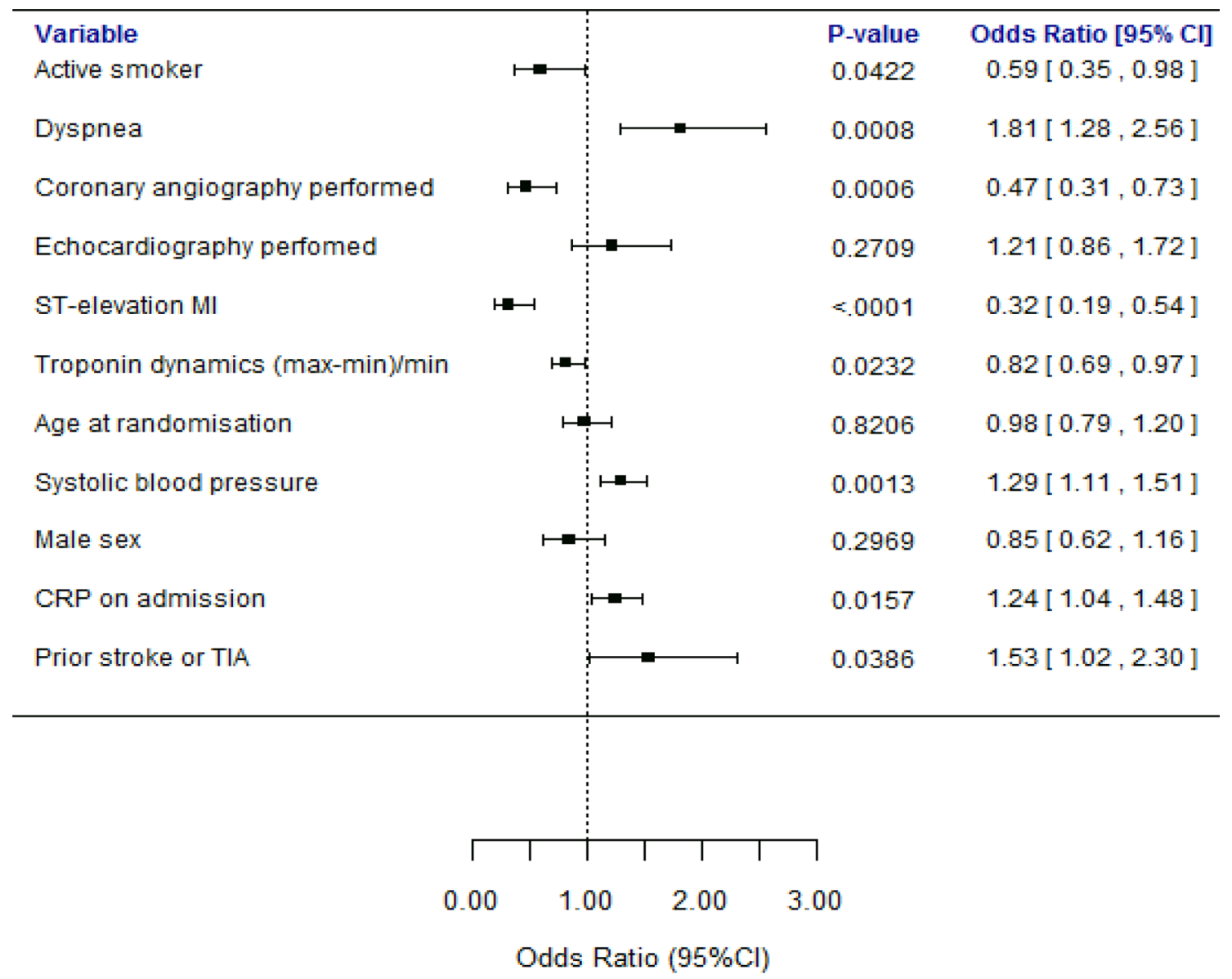

Figure 4 Multivariate logistic regression model for the effect of clinical variables on disagreement between the initial two reviewers (agreement=0). Not normally distributed numerical variables has been logarithmised and all numerical variables divided by their SD. Categorical variables are binary (yes or no). CRP, C reactive protein; MI, myocardial infarction; TIA, transient ischaemic attack.

Active smoking in a patient as well as a clear rising or falling pattern in cTn levels also makes reviewers agree more often. Some of these variables may be associated with type $1 \mathrm{MI}$, hence reflecting that reviewers agree more often when distinguishing type $1 \mathrm{MI}$ compared with when distinguishing type 2 MI or multifactorial myocardial injury. On the contrary, a high systolic blood pressure, high CRP levels on admission, symptoms of dyspnoea and a history of stroke or TIA made the reviewers disagree on the diagnosis. This is probably because these findings and anamnestic information are less specific and may apply to any of the MI or myocardial injury categories, or because they apply to the diagnosis of type $2 \mathrm{MI}$ or multifactorial myocardial injury, where reviewers disagreed more often. Regarding hypertension, opinions vary greatly on how pronounced it should be to cause myocardial ischaemia, both in previous studies ${ }^{315}$ and obviously also between reviewers in this study, indicating that some kind of guiding criteria are needed. Dyspnoea is problematic since it might be interpreted as an equivalent of angina, but has a low specificity for myocardial ischaemia. ${ }^{2}$ Elevated CRP may be associated with $\mathrm{MI}^{21}$; at the same time, it is known that sepsis may cause a secondary multifactorial myocardial injury ${ }^{22}$ but it is not certain how to interpret an elevated cTn in infections without sepsis.

Since ST elevation is almost pathognomonic for type $1 \mathrm{MI}$, it is not surprising that it is associated with a higher $\%$ agreement compared with no ST elevation; however, the kappa value was clearly lower indicating that the few cases of type $2 \mathrm{MI}$ among patients with ST elevations are especially difficult to reach consensus about.

Apart from this, the multivariate logistic regression model does not show any clinical variable associated with type $2 \mathrm{MI}$ or myocardial injury that helps reviewers agree. This further stresses that these patient groups are very heterogeneous and insufficiently defined.

\section{Limitations}

More than one third of the patients with multifactorial myocardial injury in this study were treated at a cardiology department which might have led to that cardiac-oriented causes of the injury, such as heart failure, are over-represented. Furthermore, since all patients with multifactorial myocardial injury were originally diagnosed with MI they are not representative for the total population of patients with multifactorial myocardial injury, but should rather be assumed to represent the fraction of patients with myocardial injury that are most challenging to distinguish from MI. This might partially explain both the similarities with the type $2 \mathrm{MI}$ group and the moderate rate of agreement between reviewers when differentiating between multifactorial myocardial injury and MI. However, the similarities between type $2 \mathrm{MI}$ and myocardial injury groups regarding background profile seen in this study are seen in most studies on the subject. The differences related to ischaemia between these groups are rather more clear in this study where the patients with type $2 \mathrm{MI}$ apart from 
having higher peak cTn levels and a more pronounced rising or falling pattern on serial cTn samplings had also a higher prevalence of chest pain. ${ }^{13} 14$

The retrospective design of this study may also have affected the classification done by the reviewers. There might have been clinical information not captured in the patient records that would have changed the classification in some rare cases. However, in a clinical situation the classification may be even more difficult, since it must be done in a quite early stage, often in the emergency department with substantially less available information, deciding an adequate admission strategy, handling and treatment of the patients.

\section{CONCLUSION}

The results from this study indicate that the current classification of MI and myocardial injury subtypes presented in the third universal definition of $M I$ is indistinct. Even for specially trained reviewers, with access to all clinical information retrospectively, this classification only gave a moderate rate of agreement in deciding the diagnosis, suggesting that this classification is also very challenging for a practicing clinician. Thus, there is a need for more precise and clinically applicable criteria for the current classification, especially for type $2 \mathrm{MI}$ diagnosis and myocardial injury.

\section{Key messages}

\section{What is already known on this subject?}

There are no studies investigating the interobserver agreement between physicians when differentiating between myocardial infarction (MI) and myocardial injury subtypes, strictly adhering to the classification presented in the third universal definition of MI. The interpretation of this classification varies considerably between clinicians and in different studies.

\section{What might this study add?}

There's a moderate rate of agreement ( $\kappa: 0.55)$ between trained physicians when classifying patients into $\mathrm{Ml}$ or myocardial injury subtypes according to the current universal definition. The interobserver agreement is better when distinguishing type $1 \mathrm{MI}$ (к: 0.61) compared with type $2 \mathrm{Ml}(\kappa: 0.54)$ and multifactorial myocardial injury (к: 0.48). Performing a coronary angiography makes physicians more likely to agree in this classification, while symptoms of dyspnoea and a high systolic blood pressure are associated with disagreement.

\section{How might this impact on clinical practice?}

The results of this study show that MI subtypes and myocardial injury in the current universal definition are insufficiently well defined, especially type $2 \mathrm{MI}$ and myocardial injury need to be better defined. Furthermore, the results indicate that assessing the nature of myocardial injury is particularly demanding in patients with dyspnoea and hypertension.

Acknowledgements A special thanks to all local physicians and nurses who have contributed by collecting data at Eskilstuna Hospital, Falun Central Hospital, Gävle County Hospital, Jönköping County Hospital, Karlstad Central Hospital, Danderyd Hospital and Örebro Hospital.

Contributors AG has participated in the planning, conduct and reporting of the study. BL has participated in the planning, conduct and reporting of the study. GB has participated in the conduct and reporting of the study. NH has participated in the planning (statistical advice), conduct (statistical analyses) and reporting of the study. MH has participated in the conduct and reporting of the study. KES has participated in the conduct and reporting of the study. TB has participated in the planning, conduct and reporting of the study.

Funding This study was supported by grant from the Swedish Foundation for Strategic Research. The Swedish Foundation for Strategic Research had no role in the design of the study; collection, management, analysis and interpretation of the data; preparation, review, or decision to submit the manuscript for publication. The SWEDEHEART registry is funded by the Swedish Society of Cardiology, the Swedish Society of Thoracic Radiology, the Swedish Society of Thoracic Surgery and the Swedish Heart Association. The registry is financed by the government and the Swedish Association of Local Authorities and Regions.

Competing interests None declared.

Ethics approval The study has been approved by the Regional Ethical Review Board Uppsala, reference number 2012/208.

Provenance and peer review Not commissioned; externally peer reviewed.

Open access This is an open access article distributed in accordance with the Creative Commons Attribution Non Commercial (CC BY-NC 4.0) license, which permits others to distribute, remix, adapt, build upon this work non-commercially, and license their derivative works on different terms, provided the original work is properly cited and the use is non-commercial. See: http://creativecommons.org/ licenses/by-nc/4.0/

(c) Article author(s) (or their employer(s) unless otherwise stated in the text of the article) 2018. All rights reserved. No commercial use is permitted unless otherwise expressly granted.

\section{REFERENCES}

1 Thygesen $\mathrm{K}$, Alpert JS, White HD, et al. Universal definition of myocardial infarction Kristian Thygesen, Joseph S. Alpert and Harvey D. White on behalf of the Joint ESC/ ACCF/AHA/WHF Task Force for the Redefinition of Myocardial Infarction. Eur Heart J 2007:28:2525-38.

2 Thygesen K, Alpert JS, Jaffe AS, et al. Third universal definition of myocardial infarction. J Am Coll Cardiol 2012;60:1581-98.

3 Saaby L, Poulsen TS, Hosbond S, et al. Classification of myocardial infarction: frequency and features of type 2 myocardial infarction. Am J Med 2013;126:789-97.

4 Sandoval Y, Thordsen SE, Smith SW, et al. Cardiac troponin changes to distinguish type 1 and type 2 myocardial infarction and 180-day mortality risk. Eur Heart J Acute Cardiovasc Care 2014;3:317-25.

5 Shah AS, McAllister DA, Mills R, et al. Sensitive troponin assay and the classification of myocardial infarction. Am J Med 2015;128:493-501.

6 Jernberg T, Attebring MF, Hambraeus K, et al. The Swedish Web-system for enhancement and development of evidence-based care in heart disease evaluated according to recommended therapies (SWEDEHEART). Heart 2010;96:1617-21.

7 Harnek J, Nilsson J, Friberg O, et al. The 2011 outcome from the Swedish Health Care Registry on Heart Disease (SWEDEHEART). Scand Cardiovasc J 2013;47(Suppl 62):1-10.

8 Javed U, Aftab W, Ambrose JA, et al. Frequency of elevated troponin I and diagnosis of acute myocardial infarction. Am J Cardiol 2009:104:9-13.

9 Ambrose JA, Loures-Vale A, Javed U, et al. Angiographic correlates in type 1 and $2 \mathrm{Ml}$ by the universal definition. JACC Cardiovasc Imaging 2012;5:463-4.

10 Lippi G, Sanchis-Gomar F, Cervellin G. Chest pain, dyspnea and other symptoms in patients with type 1 and 2 myocardial infarction. A literature review. Int I Cardiol 2016;215:20-2.

11 Saaby L, Poulsen TS, Diederichsen AC, et al. Mortality rate in type 2 myocardial infarction: observations from an unselected hospital cohort. Am J Med 2014; 127:295-302.

12 Baron T, Hambraeus K, Sundström J, et al. Type 2 myocardial infarction in clinical practice. Heart 2015;101:101-6.

13 Cediel G, Gonzalez-Del-Hoyo M, Carrasquer A, et al. Outcomes with type 2 myocardial infarction compared with non-ischaemic myocardial injury. Heart 2017; 103.

14 Sarkisian L, Saaby L, Poulsen TS, et al. Clinical characteristics and outcomes of patients with myocardial infarction, myocardial injury, and nonelevated troponins. Am J Med 2016;129:446.e5-446.e21.

15 Landes U, Bental T, Orvin K, et al. Type 2 myocardial infarction: a descriptive analysis and comparison with type 1 myocardial infarction. J Cardiol 2016:67:51-6.

16 Smilowitz NR, Weiss MC, Mauricio R, et al. Provoking conditions, management and outcomes of type 2 myocardial infarction and myocardial necrosis. Int J Cardiol 2016;218:196-201

17 Januzzi JL, Filippatos G, Nieminen M, et al. Troponin elevation in patients with heart failure: on behalf of the third universal definition of myocardial infarction global task force: heart failure section. Eur Heart J 2012;33:2265-71.

18 Newby LK, Jesse RL, Babb JD, et al. ACCF 2012 expert consensus document on practical clinical considerations in the interpretation of troponin elevations: a report of the American College of Cardiology Foundation task force on Clinical Expert Consensus Documents. J Am Coll Cardiol 2012;60:2427-63. 
19 McMurray JJV, Adamopoulos S, Anker SD, et al. ESC Guidelines for the diagnosis and treatment of acute and chronic heart failure 2012. Eur J Heart Fail 2012;14:803-69.

20 Landis JR, Koch GG. The measurement of observer agreement for categorical data. Biometrics 1977:33:159-74.
21 Wang J, Tan GJ, Han LN, et al. Novel biomarkers for cardiovascular risk prediction. J Geriatr Cardiol 2017:14:135-50.

22 Agewall S, Giannitsis E, Jernberg T, et al. Troponin elevation in coronary vs. noncoronary disease. Eur Heart J 2011;32:404-11. 